\title{
Student understanding of superposition: vectors and wave functions
}

\author{
Paul J. Emigh ${ }^{1}$, Gina Passante ${ }^{2}$, and Peter S. Shaffer ${ }^{1}$ \\ ${ }^{1}$ Department of Physics, University of Washington, Seattle WA 98195-1560 \\ ${ }^{2}$ Department of Physics, California State University, Fullerton, Fullerton CA 92834-6900
}

\begin{abstract}
As part of a broad investigation of student understanding in physics, we have examined student ability with superposition throughout introductory and upper-division courses in physics. This research has focused on examining student ability to add and subtract vector quantities and the wave functions associated with quantum physics. We present results from a series of research tasks designed to probe student understanding of superposition in each of these contexts at various points in undergraduate instruction. In addition, we describe and discuss certain patterns in student reasoning that have been identified across the different tasks, contexts, and courses.
\end{abstract}

\section{INTRODUCTION}

Vectors are an important part of physics instruction at both the introductory and the upper-division levels. Past research has shown that many introductory physics students have difficulty with concepts such as superposition and addition of vectors in a wide variety of contexts [1-5]. Instruction on topics such as classical waves (e.g., waves on a string or EM waves) and quantum mechanics also rely on superposition. Prior research has also found that students struggle with superposition in each of these contexts [6-10].

In this paper, we identify patterns in student reasoning about superposition across some of these different contexts. In particular, we focus on students' qualitative ability to add and subtract vectors and wave functions. First, we describe the context and background for this research. Then, for each context (vectors and wave functions), we present the tasks used to probe student ideas and identify those lines of reasoning that appear across a wide variety of contexts. Lastly, we discuss how the similarities in student thinking might be used to inform the development of curriculum for improving student understanding.

\section{CONTEXT FOR RESEARCH}

This study was conducted at the University of Washington (UW) with students from the introductory calculus-based physics sequence and from sophomore- and junior-level quantum courses for physics majors. In each course, we gave qualitative research tasks asking students to compare two cases in which either vectors or wave functions were superposed. Each task asked students to explain their reasoning. All tasks were given after students had covered any relevant material in lecture. Each task that focused on vectors was given online, and students received credit whether or not they gave correct answers. Tasks involving wave functions were part of midterm exams.
This investigation is grounded in the methods and practices of the Physics Education Group at UW [11]. In particular, we used a wide variety of tasks to examine student understanding from different perspectives by frequently making changes to wording, structure, and/or context. As a result, the tasks used in this study are not isomorphic, and we do not directly compare the quantitative results across different versions. Instead, we focus on examining the lines of reasoning in student explanations. Then, we compare the results across different versions and contexts to identify common patterns. This research can then inform curriculum targeted at those student ideas that are most prevalent and persistent.

\section{VECTORS}

We have used many different tasks to probe student understanding of vector superposition over many years and many different courses. Each reveals different aspects of student thinking about superposition. One such task shows students two pairs of vectors (see Fig. 1) that all have the same length. The vectors are given as generic mathematical objects, without any accompanying physical context. Students are asked to determine whether the magnitude of the vector sum $\mathbf{A}+\mathbf{B}$ is greater than, less than, or equal to the magnitude of the vector sum $\mathbf{C}+\mathbf{D}$.

This task can be answered by noting that vectors $\mathbf{A}$ and $\mathbf{B}$ are closer to being parallel than vectors $\mathbf{C}$ and $\mathbf{D}$. Thus, there is less cancellation between $\mathbf{A}$ and $\mathbf{B}$ than there is between $\mathbf{C}$ and $\mathbf{D}$, so the magnitude of the sum is greater for the former. Several alternate lines of reasoning were considered correct, such as algebraic solutions using cancellation between vector components or algorithms for vector addition such as the tip-to-tail method.

This task was administered to more than 500 students during the first week of introductory physics at UW, after instruction on vectors. Variants of the task (e.g., versions 


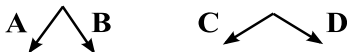

FIG 1. Four equal-length vectors shown to students as part of a task on vector superposition. Students were asked to compare the sum of each pair.

with a gridded background and/or different angles) were also given to more than 500 students. The tasks were given in courses with different instructors over many quarters. The percentage who answered correctly has varied between $62 \%$ and $76 \%$. About half of those who answered correctly gave correct reasoning (see above); the other half gave incomplete explanations (e.g., many students cited "vector addition" with no further reasoning). These results, and the common incorrect lines of reasoning discussed below, are similar to what has been reported elsewhere [1].

No incorrect answer was strongly preferred. Students who answered "less than" $(\mathbf{A}+\mathbf{B}<\mathbf{C}+\mathbf{D})$ gave explanations that tended to correspond to a method for subtracting each pair of vectors instead of adding them. Some stated that a larger angle between the vectors directly corresponds to a larger sum. Others gave very brief answers, often similar to the incomplete explanations given in support of the correct answer discussed above.

Students who answered "equal to" $(\mathbf{A}+\mathbf{B}=\mathbf{C}+\mathbf{D})$ predominantly justified their answer by stating that the sums are equal because the magnitudes of all vectors are equal. This explanation suggests that these students are adding vectors as if they were scalars. A few students specifically noted that the relative direction of the vectors does not impact the magnitude of their sum.

The task discussed above was designed to probe student thinking about vectors in a generic context as a means for providing insight into student difficulties with vectors in physics contexts (e.g., velocities or forces). We have also administered versions of this task based on superposition of vectors in various physical contexts throughout introductory physics. Example tasks for force and momentum are shown in Fig. 2. Additional tasks in contexts such as velocity, electric or magnetic force or field, and universal gravitation have also been given. The tasks are designed to focus on the same underlying concept (superposition) but are not intended to be identical. The differences are intended to help broaden our insight into student thinking on this topic.

The overall results have varied somewhat from context to context. In general, fewer students gave the correct answer to tasks involving a physical context (between $35 \%$ and $57 \%$ ) than those involving a generic context. The most common incorrect answer was universally that the sums in the two cases are equal (between $33 \%$ and $54 \%$ of the students). The percentage choosing "less than" was between $4 \%$ and $18 \%$. Since these tasks involve additional reasoning steps related to the underlying physics, it is not surprising that they are more difficult for students.

Despite the variable percentages, the lines of reasoning given in support of each answer did not differ substantially from context to context. Students who answered correctly

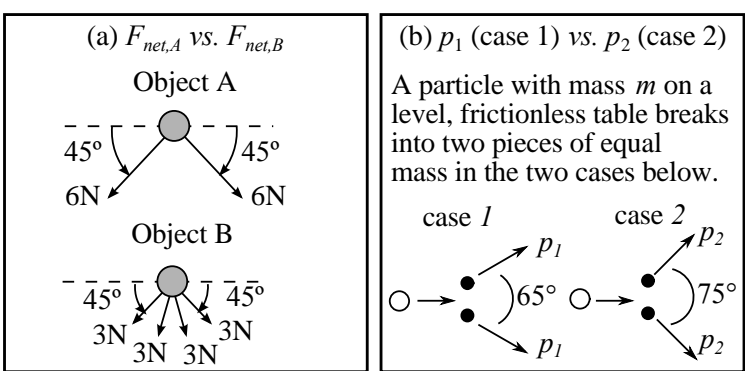

FIG 2. Tasks designed to probe student thinking about vectors in the physics contexts of (a) forces and (b) momenta. In each task, students are asked to compare the magnitudes of two quantities.

gave either correct or incomplete reasoning, but rarely gave explanations that were entirely incorrect. Students who chose "equal to" (the predominant incorrect answer) based their answer on the equivalence of the magnitudes of the vectors to be added. Students who answered "less than" tended to give vague explanations, often indicative of using an incorrect algorithm or of an algebraic sign error.

\section{WAVE FUNCTIONS}

In order to probe student ideas about superposition in quantum mechanics, we designed tasks that require students to add wave functions with different relative phases. In these tasks, we varied relative phase in the same way that relative angle was varied when we asked students about vector superposition. Each task presented students with a pair of wave functions, such as those shown in Fig. 3. Then, students were told to consider a specific linear combination of these two wave functions. A variety of different linear combinations have been used; in this paper, we discuss results from versions that used either $\Psi_{A}=\frac{1}{\sqrt{2}}\left(\psi_{1}+i \psi_{2}\right) \quad$ or $\quad \Psi_{B}=\frac{1}{\sqrt{2}}\left(\psi_{1}-\psi_{2}\right)$. In each case, the students were asked if the probability of measuring the particle to the left of the origin is greater than, less than, or equal to the probability of measuring the particle to the right of the origin.

To answer this task, the probability can be determined by integrating the probability density (the modulus square of the wave function) on either side of the origin. The two probabilities can be compared graphically by examining the corresponding areas under the probability density curve. For $\Psi_{A}$, the probabilities are equal because there is neither

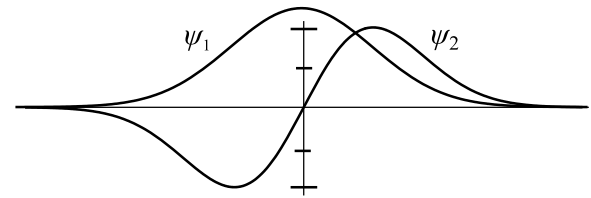

FIG 3. An example of the two wave functions (one symmetric and one anti-symmetric) given as part of a task on superposition in quantum mechanics courses. 
constructive nor destructive interference between $\psi_{1}$ and $\psi_{2}$, since the relative phase is $\pi / 2$. For $\Psi_{B}$, there is constructive interference on the left and destructive interference on the right, so the area and the probability on the left are greater.

The percentage of the students who gave each answer varied depending on population-introductory (honors), sophomore, or junior - and on whether they were asked about $\Psi_{A}$ or $\Psi_{B}$. Between $50 \%$ and $80 \%$ gave the correct answer, many with explanations that were incorrect or incomplete, especially for $\Psi_{A}$. For example, as many as one-third of student explanations to the $\Psi_{A}$ version were based on the symmetries of $\psi_{1}$ and $\psi_{2}$, without any reference to addition or superposition. It was common for students who were asked about $\Psi_{B}$ to use identical reasoning, in which case the probabilities are not equal.

For the $\Psi_{A}$ tasks, almost all students who answered incorrectly stated that the probability on the left side of the origin is less than the probability on the right side. These students' explanations predominantly indicate that they were summing $\psi_{1}$ and $\psi_{2}$ directly, without taking into account the impact of the relative imaginary phase between the wave functions. Responses that ignored the given phase difference were also present for the $\Psi_{B}$ tasks (between $16 \%$ and $24 \%$ ), which roughly corresponds to adding the two wave functions instead of subtracting them.

\section{PATTERNS IN STUDENT REASONING}

There are some commonalities in the lines of reasoning across the different tasks discussed above. In particular, we have identified three general patterns that appear across the different contexts that we have investigated. These are summarized in Table I, with representative sample quotes. The patterns are not necessarily mutually exclusive, and it was not uncommon for some responses to show elements of more than one. It should also be recalled that the wave function task requires more sophisticated reasoning than the vectors task, which limits our ability to draw more specific conclusions than those outlined below.

The most widespread incorrect line of reasoning was that the magnitude of a vector sum depends only on the magnitudes of the constituent vectors, and not on the relative angle between them (or, in the case of waves, on the phase difference between them). For example, students often appeared to treat vectors as if they were scalars, attending to magnitude but ignoring direction altogether. This line of reasoning was especially common for vectors that represent physical quantities (like forces). It also appeared in the responses to all wave function tasks. Some students even explicitly stated that either direction (for vectors) or negative/imaginary phase (for wave functions) does not matter when determining a final answer, though this was not the case for all students in this category.

Because this reasoning was so common, we designed a more targeted task to investigate it in more detail. In this new task, we asked students which of the following piece(s) of information are sufficient to determine the magnitude of the sum of two vectors that lie in the $x y$-plane: (1) the magnitude of the first vector, (2) the magnitude of the second vector, and (3) the angle between the two vectors. Students were told to select all applicable answers, or to indicate that none of these pieces are necessary. Part of the goal of this task was to explore any differences between students' knowledge about vectors in general and the ways in which they answer tasks that involve actual vectors.

This task was given at the start of introductory physics, after instruction on vectors, to more than 500 students. Only $49 \%$ correctly identified that all three pieces of information are necessary to determine the magnitude of the vector sum. A substantial fraction of the students $(30 \%)$ specified that the angle between the two vectors is not necessary (but that the magnitudes are). The explanations given by these students explicitly confirmed that they believe the angle is not necessary. Often, students justified this answer by noting that the question asks only about the magnitude of the vector sum, not about the direction of the vector sum. These results are consistent with the idea that angle, direction, sign, and/or phase is irrelevant, as was common in the responses to the tasks described above.

A possibly related pattern involves apparent confusion between addition and subtraction. For example, students often described a method that would be correct for

TABLE I. Common patterns in student reasoning about superposition across different contexts.

\begin{tabular}{|c|c|c|}
\hline \multirow{2}{*}{$\begin{array}{c}\text { Description of pattern } \\
\text { in student reasoning }\end{array}$} & \multicolumn{2}{|c|}{ Example student explanations } \\
\hline & Vectors (generic context) & Wave Functions ( $\Psi_{B}$ version) \\
\hline $\begin{array}{l}\text { Attending primarily to } \\
\text { magnitude (not to relative } \\
\text { direction or phase) }\end{array}$ & $\begin{array}{l}\text { "Equal to. If the vectors are the same } \\
\text { lengths then the magnitude of the resultant } \\
\text { of each pair will be the same." }\end{array}$ & $\begin{array}{l}\text { "Equal to. Negative doesn't matter, } \\
\text { calculating probabilities squares away } \\
\text { negative portions of [the] function." }\end{array}$ \\
\hline $\begin{array}{l}\text { Adding instead of subtracting, } \\
\text { or vice versa }\end{array}$ & $\begin{array}{l}\text { "Less than. When you line the vectors up } \\
\text { [tail to taill and connect the ends with a } \\
\text { line, }[C+D \text { 's line] would be greater." }\end{array}$ & $\begin{array}{l}\text { "Less than. The amplitudes add on the } \\
\text { right whereas on the left they oppose, } \\
\text { making the amplitude smaller on the left." }\end{array}$ \\
\hline $\begin{array}{l}\text { Tendency to use incomplete } \\
\text { explanations that did not } \\
\text { make use of superposition }\end{array}$ & $\begin{array}{l}\text { "Greater than. The angle between } \\
\text { [the] two vectors is smaller." }\end{array}$ & $\begin{array}{c}\text { "Equal to [ } \Psi_{A} \text { version]. Because while } \psi_{1} \\
\text { is symmetric and } \psi_{2} \text { is anti-symmetric, } \\
\text { both }\left|\psi_{1}\right|^{2} \text { and }\left|\psi_{2}\right|^{2} \text { are symmetric." }\end{array}$ \\
\hline
\end{tabular}


subtracting two quantities when they were asked to add them, or for adding when they were asked to subtract. In some cases, this kind of reasoning may be related to the previous pattern, since it also represents a failure to account for the relative direction. Furthermore, the fact that this kind of error persists from introductory through upperdivision physics suggests that it should not be ignored.

Another pattern that we have identified is that students often gave brief, incomplete explanations that omit key elements of a complete solution. For example, some responses to the vectors task consisted of statements like "vector addition" and no further explanation. Often, students gave no indication in their explanations that they made use of superposition in answering. This was true of explanations given in support of both correct and incorrect answers, which makes it difficult to claim anything certain about the fundamental conceptual understanding of students whose explanations are too brief to characterize.

Lastly, it should be noted that some of the lines of reasoning that students use do not fit within any of the patterns that we have described here. It was not uncommon for students to base their answer on the surface features of any given task, or on the context used (e.g., when we asked about momentum vectors, some explanations relied heavily on "conservation of momentum"). These incorrect lines of reasoning typically do not appear to be directly related to the use of superposition, and have often been identified in previous studies on student understanding of the relevant physics topic. In particular, there were several alternate (i.e., non-superposition-based) lines of reasoning associated with the wave function tasks. This is not necessarily surprising, as many of the concepts in quantum mechanics that students find difficult are relevant to the tasks discussed throughout this paper [10]. Some of these are identified elsewhere in the research literature on student understanding of quantum mechanics, including the incorrect idea that a quantum superposition can be considered as a mixture between its constituent states, such that it is actually one or the other, and not both [9].

\section{IMPLICATIONS}

An important goal of this investigation is to help inform the teaching of physics. The similarity in student reasoning

[1] N. Nguyen \& D. Meltzer, Am. J. Phys. 71 (6), 630 (2003).

[2] P.S. Shaffer \& L.C. McDermott, Am. J. Phys. 73 (10), 921 (2005).

[3] A.F. Heckler \& T.M. Scaife, Phys. Rev. ST Phys. Educ. Res. 11, 010101 (2015).

[4] P. Barniol \& G. Zavala, Phys. Rev. ST Phys. Educ. Res. 10, 020115 (2014).

[5] S. Flores et al., Am. J. Phys. 72, 460 (2004). across so many contexts has implications for introductory and quantum physics instruction. For example, in a typical year-long introductory physics sequence, students might encounter superposition in 5-10 distinct contexts. Upperdivision courses in physics might easily match that number. Even after instruction that encompasses multiple topics, the difficulties that students have tend to be similar across both different contexts and different levels of instruction.

It is thus possible that instruction at the introductory level might be improved by promoting student attentiveness to the similar principles that appear throughout their courses on physics. Students can be guided to identify and employ correct lines of reasoning in both simple and challenging contexts. They might also benefit from explicitly confronting the most common incorrect lines of reasoning associated with superposition-not just when vectors are first introduced, but each time they occur in a new context.

The same strategy could also prove useful at the upperdivision level. Students at that level are generally expected to have mastered superposition in introductory contexts. These students' knowledge could then be leveraged to help them recognize and reflect on the similarities between vector and wave function addition, especially if similar exercises had already been employed throughout their introductory coursework. This might then allow students to engage more directly with other challenging concepts, such as those that are unique to quantum mechanics.

The elements of student understanding explored in this paper are, in essence, instances of transfer. We believe that a theoretical framework such as Transfer in Pieces [12] might be productive in further analyzing and interpreting student ideas about superposition, and in developing curriculum to address those ideas.

\section{ACKNOWLEDGEMENTS}

We would like to thank the many instructors who welcomed this research into their classrooms, in particular John Rehr and Daryl Pedigo. We also thank the work of several undergraduate students who aided in analysis of the data. This work has been supported in part by the National Science Foundation under Grant No. DUE-1022449.

[6] M. Wittmann, Ph.D. thesis, U. of Maryland (1998).

[7] K. Wosilait, Ph.D. thesis, U. of Washington (1996).

[8] M. Kryjevskaia et al., Am. J. Phys. 79, 508 (2011).

[9] G. Passante et al., Phys. Rev. ST Phys. Educ. Res. 11, 020111 (2015).

[10] P.J. Emigh, Ph.D. thesis, U. of Washington (2015).

[11] P. Heron, at PERC 2003, Madison, WI (2003).

[12] J. F. Wagner, Cogn. Instruct, 24, 1 (2006). 